\title{
O complexo produtivo da saúde e sua articulação com o desenvolvimento socioeconômico nacional
}

Laís Silveira Costa, Carlos Augusto Grabois Gadelha, José Maldonado, Marcelo Santo e Antoine Metten

\section{Introdução}

O protagonismo da saúde na agenda de desenvolvimento do Brasil tem sido amplamente reconhecido e valorizado. Isso decorre do fato de que políticas e ações de saúde, além de proporcionar o bem-estar da população, apresentam benefícios que extrapolam a especificidade do setor, a exemplo de seu impacto na geração de renda e emprego nacionais.

Não bastassem essas qualidades, o papel estratégico da saúde no processo de desenvolvimento é realçado por articular um sistema produtivo, de base industrial (química, biotecnológica, mecânica, eletrônica e de materiais) e de serviços, de forma interdependente e sistêmica, designado como complexo econômicoindustrial da saúde (CEIS) ${ }^{1}$. O arcabouço conceitual do CEIS reconhece tanto a importância social da saúde, como também sua função enquanto produtora de insumos e produtos para a prestação de serviços essenciais, pontuando seu caráter sistêmico. 
Nesse contexto, que relaciona a lógica produtiva e social, o complexo da saúde é capaz de apresentar oportunidades para a superação da tensão observada entre as dimensões econômicas e sócio-sanitárias, uma vez mediados os interesses conflitantes envolvidos na saúde.

Não obstante a importância do CEIS para o desenvolvimento nacional e o seu potencial para estabelecimento de uma relação virtuosa entre os interesses diversos no campo da saúde (VIANA; NunES; SiLVA, 2011), ainda persiste uma visão limitada do seu caráter sistêmico, conforme enfatizam Gadelha et al. (2012). Essa limitação tem implicações negativas no desenvolvimento da base produtiva nacional com reflexos insatisfatórios para a saúde coletiva e para o desenvolvimento econômico, observados no crescente déficit da balança comercial da saúde.

Tal realidade pontua desafios e ameaças à manutenção de um sistema universal do porte do brasileiro, em especial ao se considerar a transição demográfica e os custos crescentes da saúde. Cabe observar que, sem a superação da fragilidade da base produtiva, persistirão obstáculos à oferta universal de bens e serviços, acentuando a vulnerabilidade do sistema de saúde (GAdelha e Costa, 2013).

A relevância desse estudo deriva da percepção de que somente avançando no conhecimento sobre essa base produtiva poder-se-á aprimorar o entendimento sobre os desafios que se apresentam para a efetividade do sistema nacional de saúde e, adicionalmente, qualificar a elaboração e implementação de políticas públicas para o fortalecimento do complexo da saúde.

Dito isso, este artigo objetiva aprofundar o conhecimento sobre o CEIS e a dinâmica de seus subsistemas, com o intuito de melhor entender os processos inovativos da saúde. Vale notar que, ao considerar a institucionalidade do CEIS, que é, a um só tempo, pública e privada, adota-se neste artigo uma abordagem sistêmica propiciada tanto pelo arcabouço da economia política, que envolve o estudo das relações sociais de produção e de acumulação de capital, quanto pelo instrumental teórico dos sistemas de inovação (SI), que entende o caráter essencialmente social dos processos de geração de inovação (FreEman, 1987; Lundvall, 1988; NeLson, 1993).

Além desta introdução, o texto estrutura-se a partir da relação estabelecida entre saúde e desenvolvimento e do detalhamento dos subsistemas do complexo econômico-industrial da saúde. Contextualiza também a relevância do sistema nacional de inovação em saúde, de forma a tornar sustentável o sistema de saúde como um todo. Por fim, ressalta a importância do desenvolvimento da base produtiva e inovativa da saúde no País, de modo a atender às necessidades sociais, e, nesse contexto, enfatiza a influência do Estado nessa arena política.

\section{O complexo da saúde na agenda de desenvolvimento nacional}

Este artigo analisa a saúde sob um olhar sistêmico que abrange, além da sua dimensão social, seu contexto de produção. Para isso, o recorte de análise é o complexo econômico-industrial da saúde, uma vez que esse articula simultaneamente variáveis sociais, econômicas e inovativas, questões-chave no atual cenário mundial de globalização assimétrica e competitiva.

Adota a perspectiva teórica de Furtado (1964), para quem o desenvolvimento representa "um processo de mudança 
social pelo qual o crescente número de necessidades humanas (...) são satisfeitas através de uma diferenciação no sistema produtivo, gerado pela introdução de inovações tecnológicas" (FURTADO, 1964, p. 27). Ou seja, trata de uma concepção de desenvolvimento diferenciada que reconhece a influência de variáveis econômicas e inovativas no bem-estar coletivo.

Ademais, chama-se atenção para o fato de que, na sociedade contemporânea do conhecimento, a inovação merece destaque, uma vez que se torna um diferencial estratégico na definição do posicionamento de ordem econômica e produtiva de um país diante dos demais, exercendo, inclusive, forte influência nas condições de vida das populações.

Para Furtado (1998), um modelo de desenvolvimento ideal deveria interromper a reprodução dos padrões de consumo das minorias privilegiadas e buscar a satisfação das necessidades fundamentais do conjunto da população (FURTAdo, 1998). Nesse sentido, a inovação tecnológica deveria ser reorientada para a busca do bem-estar coletivo.

Seguindo essa perspectiva, pode-se aferir que o desenvolvimento de um país encontra-se intimamente relacionado às oportunidades disponibilizadas à sua população com vistas ao seu bem-estar, reafirmando-se a necessidade tanto de subsidiar quanto de orientar a indução da taxa de progresso técnico, para que as novas tecnologias sigam rumos socialmente desejáveis e sustentáveis (COSTA et al., 2012), chamando atenção para o fato de que um determinado padrão de desenvolvimento tecnológico representa um modelo particular de sociedade, e vice-versa.

Parte-se do reconhecimento de que o “desenvolvimento tecnológico não é neutro" e que é estabelecida uma dupla relação de causalidade, em que tanto a orientação do desenvolvimento tecnológico influencia um determinado padrão de sociedade, como a orientação socioeconômica e as institucionalidades influenciam o desenvolvimento tecnológico de uma determinada nação (TigRe, 2006).

Esse enfoque nos remete à pertinência do uso de uma abordagem estruturalista do campo da economia política e do

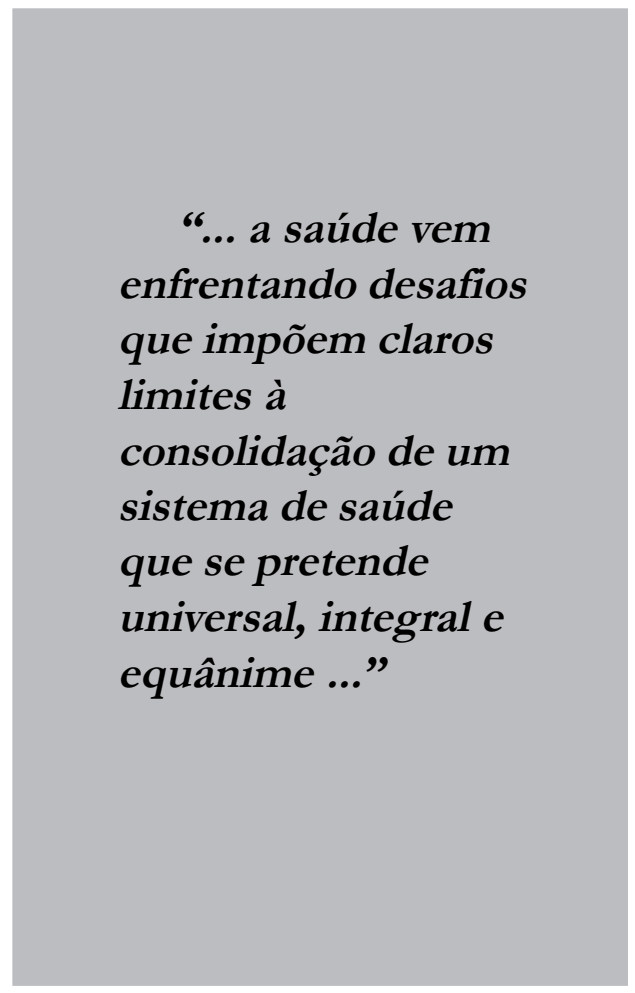

arcabouço teórico dos sistemas de inovação, uma vez que processos de inovação são, por natureza, contextualizados socialmente em economias complexas do capitalismo (FrEEMAN, 1987; Soete; Verspagen; Weel, 2010). Ademais, esses enfoques reconhecem o papel dos diversos atores, de suas interações e interesses, que levam à 
conformação dos sistemas de saúde, bem como sua importância para a conformação de um modelo nacional de desenvolvimento.

Entre os trabalhos desenvolvidos no campo da saúde utilizando essa linha teórica vale mencionar algumas análises. Viana e Elias (2007) reconhecem a possibilidade de combinar crescimento econômico, mudanças na estrutura produtiva e melhora do padrão de vida da população. Machado et al. (2008) chamam atenção para a relevância do estudo da relação entre saúde e desenvolvimento, necessária para instrumentalizar os grandes objetivos da reforma sanitária. Gadelha (2007) alerta para a inadequação de uma abordagem que desconhece o caráter sistêmico da base produtiva da saúde. A desarticulação histórica entre esses elementos que conformam os sistemas de saúde apresenta impactos deletérios para a instituição do modelo de bem-estar social, conforme preconizado na Carta Magna de 1988.

A promulgação da Constituição Federal de 1988 (CF/88) realça e consolida no Brasil a interdependência mútua entre saúde e desenvolvimento. Ao estabelecer que a saúde é um direito de todos e um dever do Estado, a CF/ 88 consagroua como um elemento estruturante do Estado de Bem-Estar, expressando de forma inequívoca sua importância como parte inerente ao processo de desenvolvimento. Acolhem-se, assim, os anseios do movimento reformador da saúde, especialmente quanto à visão ampliada da saúde, entendida como um direito de cidadania que, em termos gerais, significa condições dignas de vida, acesso igualitário e universal às ações e serviços de promoção, proteção e recuperação da saúde em todos os seus níveis (PAIM, 1997).
No entanto, a promulgação da CF/88 não foi suficiente para impulsionar o Sistema Único de Saúde (SUS). A concretização da saúde como um direito fundamental exige ainda um grande esforço, especialmente no que se refere ao acesso igualitário a bens e serviços de saúde para toda a população.

Mendes (1996) ressalta que, no Brasil, a instituição do modelo de bem-estar e a universalização da saúde ocorreram na contramão da história, dado que coincidiram com a adoção do modelo hegemônico neoliberal que, entre outras diretrizes, pregava a diminuição da participação do Estado.

Nesse contexto, Gadelha (2006) aponta que o processo que culminou com a criação do SUS não considerou que um sistema de saúde que se pretenda universal é composto não somente pela demanda social por bens e serviços de saúde, mas também por organizações voltadas à provisão desses serviços, pressupondo o fortalecimento de sua base produtiva. A retração da participação do Estado nas políticas industriais, assim como a abertura abrupta do mercado brasileiro, característicos da década de 1990 no Brasil (Gadelha; Maldonado; Costa, 2012), tiveram efeitos devastadores para a base industrial da saúde 2 . De acordo com Manfredini (2006, p. 161), "uma série de produtos que havia sido incorporada à produção local nas décadas anteriores deixa de ser produzida no País, como, por exemplo, marcapassos implantáveis, aparelhos de laboratório mais complexos e equipamentos radiológicos".

Considerando especificamente o impacto desse contexto no estágio de desenvolvimento do CEIS, Viana e Elias (2007) também identificam contradições no que tange à desmercantilização do acesso à saúde que pautou o aumento da demanda, 
concomitante à adoção de um modelo político liberal que afastou o Estado nacional de sua função de indutor e mediador da produção industrial da saúde e dos interesses envolvidos na mesma.

Extrapolando a análise do CEIS, Faveret e Oliveira (1990) destacam que a ausência ou inadequação de investimentos indutores de inovação tecnológica motivou o Estado brasileiro a transferir parte de sua responsabilidade com o sistema de saúde recém-criado ao setor privado, polarizando ainda mais as dimensões sociais e econômicas envolvidas no bojo da saúde.

Sobre os interesses públicos e privados no âmbito dessa agenda, Ugá e Marques (2005) defendem a tese de que o SUS foi criado em um momento em que o setor privado já se encontrava consolidado no País. Essa linha de argumento corrobora a percepção de que essa resignação do Estado, ou seja, a diminuição de seu protagonismo explica, mesmo que não integralmente, o fato de que ainda não se alcançou no Brasil o círculo virtuoso entre saúde e desenvolvimento. Questão essa preocupante no que se refere tanto ao atendimento das demandas de saúde da população quanto à inserção competitiva internacional; ainda mais quando se consideram as fragilidades enfrentadas por um país do porte do Brasil e com o modelo institucional do SUS.

Ainda que iniciativas diversas estejam sendo empreendidas visando à superação desse quadro via o fortalecimento do CEIS, a saúde vem enfrentando desafios que impõem claros limites à consolidação de um sistema de saúde que se pretende universal, integral e equânime, sugerindo a necessidade de se aprofundar o conhecimento sobre a dinâmica desse complexo. A ideia é elevar a capacidade de análise para subsidiar a formulação de políticas públicas capazes não somente de lograr competitividade internacional, mas também de mediar os conflitos entre interesses econômicos e sócio-sanitários envolvidos na agenda de saúde. Desse modo, espera-se minimizar a vulnerabilidade do sistema de saúde, assim como promover o crescimento sustentado nacional.

\section{A base produtiva da saúde}

O complexo econômico-industrial da saúde (Figura 1) é caracterizado como um aglomerado de atividades econômicas inseridas em um contexto institucional cujos segmentos industriais são responsáveis pela produção de insumos e produtos que vão confluir para a base produtiva envolvida na prestação de serviços de saúde.

Seu caráter estratégico é crescentemente relacionado ao fato de que o CEIS é intensivo em inovação. Tanto no Brasil quanto no mundo, esse complexo constitui o segundo setor mais intensivo em atividades inovativas, respondendo por mais de $20 \%$ do esforço em pesquisa e desenvolvimento mundial (GLOBAL BOOZ AND COMPANY's, 2012). Além disso, articula tecnologias de uso dual (Rundvalt e Albuquerque, 2012) que, apesar de voltadas para um determinado setor, apresentam repercussões potenciais sobre a atividade econômica como um todo, como é o caso da nanotecnologia, biotecnologia, entre outros cujo potencial de aplicação a outros setores da economia é cada vez mais evidente.

Esse complexo produtivo diferenciase, ademais, por envolver um arranjo institucional público e privado e se destaca pelo caráter sistêmico, uma vez que reconhece não somente a demanda da sociedade por bens e serviços de saúde, como também uma estrutura responsável pelas ofertas dos mesmos. 


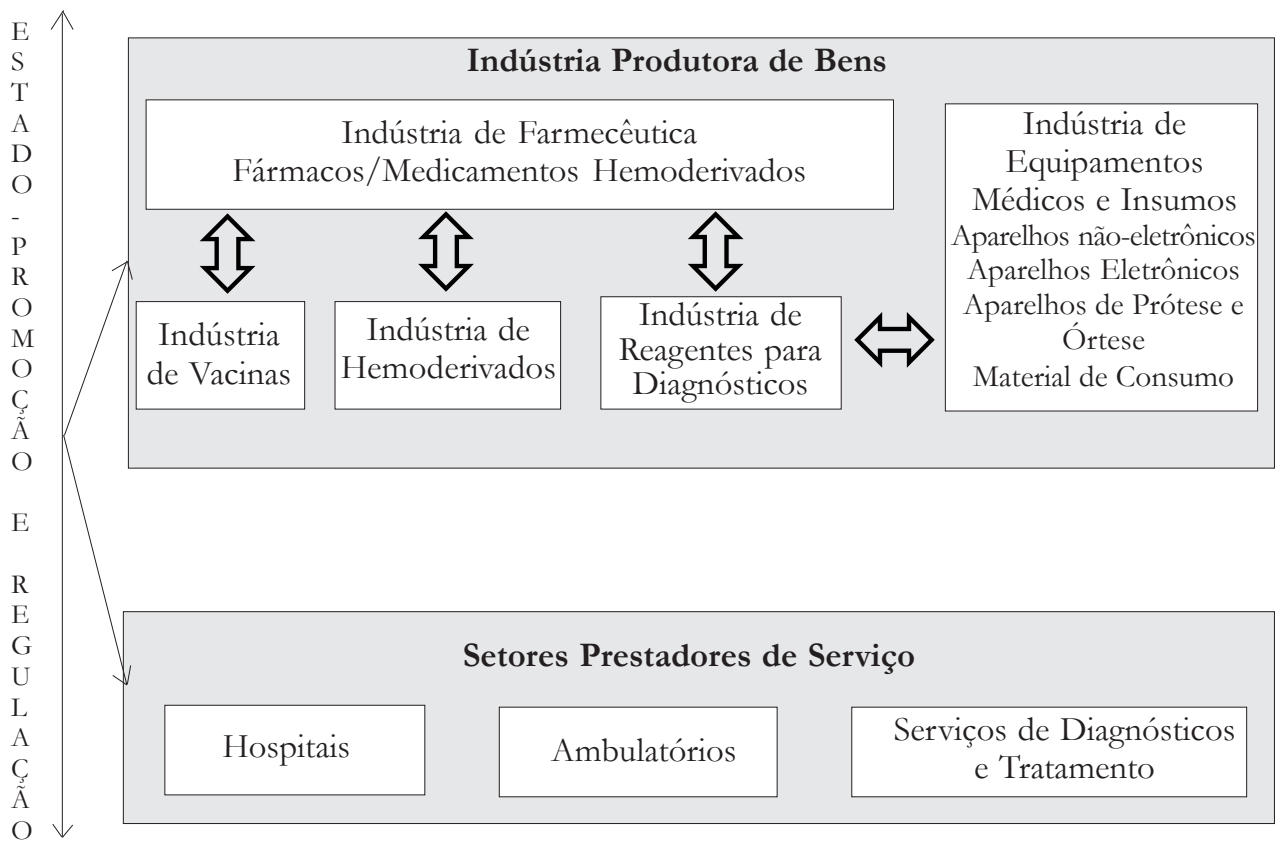

Fonte: Gadelha, 2003.

Figura 1: Morfologia do complexo econômico-industrial da saúde

Ademais, ao relacionar as dimensões social e produtiva, o CEIS apresenta potencial para a superação da dicotomia observada entre a lógica econômica e a sanitária, no que se refere às políticas para o desenvolvimento nacional. É pertinente observar que as estratégias tecnológicas em países líderes, no âmbito internacional, vêm passando por um processo de profundas transformações estruturais no que tange ao seu padrão de competitividade (Gadelha; Quental; Fialho, 2003). Essas transformações permitem tecer algumas constatações no que se refere ao complexo da saúde.

Uma dessas constatações é que, nos segmentos intensivos em tecnologia do CEIS, o espaço para pequenas empresas de base tecnológica, que não se associem ou tenham parcerias para superar as barreiras econômicas, tecnológicas e regulatórias vigentes no mercado global, é bastante reduzido (GADELHA et al., 2012). Outra é a verificação de evidências indicando que empresas líderes estão interessadas em aproveitar as oportunidades que se abrem em economias emergentes que apresentam potencial de crescimento sustentado $^{3}$, a exemplo dos BRICS (Brasil, Rússia, Índia, China e África do Sul) (GADElHA et al., 2012). Por último, tal como se evidencia nos diversos programas de políticas públicas voltados para o fortalecimento do CEIS, os Estados nacionais reconhecem que o complexo está se tornando um espaço competitivo e que, no Brasil, inicia-se a busca de uma intervenção sistêmica entre a sua base industrial e de serviços.

Tais constatações justificam a necessidade de se adensar o conhecimento sobre essa base produtiva, para melhor compreender os desafios e as oportunidades colocadas ao complexo e qualificar a ação 
pública voltada para o setor. Isso implica, por sua vez, conhecer as características e a dinâmica da cada um de seus subsistemas.

\section{A indústria de base química e biotecnológica da saúde}

O subsistema de base química e biotecnológica é composto pelas indústrias farmacêuticas e por aquelas responsáveis pela produção de vacinas, hemoderivados e reagentes para diagnóstico. Esse subsistema agrega um conjunto de segmentos produtivos que se destacam tanto por sua importância econômica, como por sua relevância no domínio de novas tecnologias em áreas estratégicas para o País (VARGAS et al., 2010). Entre os subsistemas do CEIS, o de base química e biotecnológica constitui o mais dinâmico em termos de geração e difusão de inovação. (GAdelHA et al. 2012).

Por sua vez, vale notar que a indústria farmacêutica lidera a dinâmica competitiva do subsistema e se caracteriza por ser o principal segmento de geração e difusão de inovações de base química e biotecnológica. Marcado por elevado grau de internalização da produção e intensa concentração de mercado, o segmento farmacêutico pontua a existência de interesses diversos, além dos sanitários, relacionados ao seu processo produtivo de tecnologias estratégicas, como a nanotecnologia, biotecnologia e química fina (Angell, 2007). Tanto Temporão (2002) quanto Gadelha (2002) chamam atenção para o acirramento competitivo do subsistema de base química e biotecnologia, em que as empresas líderes farmacêuticas invadem e submetem os demais segmentos às suas estratégias de inovação. Dados do IMS Health ${ }^{4}$ mostram que a concentração de mercado no segmento farmacêutico encontra-se adstrita a dez grandes empresas que, no total de suas vendas, somaram quase a metade de todo o mercado farmacêutico mundial em $2010^{5}$. Essa liderança decorre de duas características presentes em suas estratégias competitivas, quais sejam: barreiras a novos entrantes no mercado, devido aos vultosos investimentos em P\&D e marketing necessários, e o monopólio temporário mediante patentes, operando sobre produtos cuja demanda é particularmente inelástica (GADELHA et al. 2012).

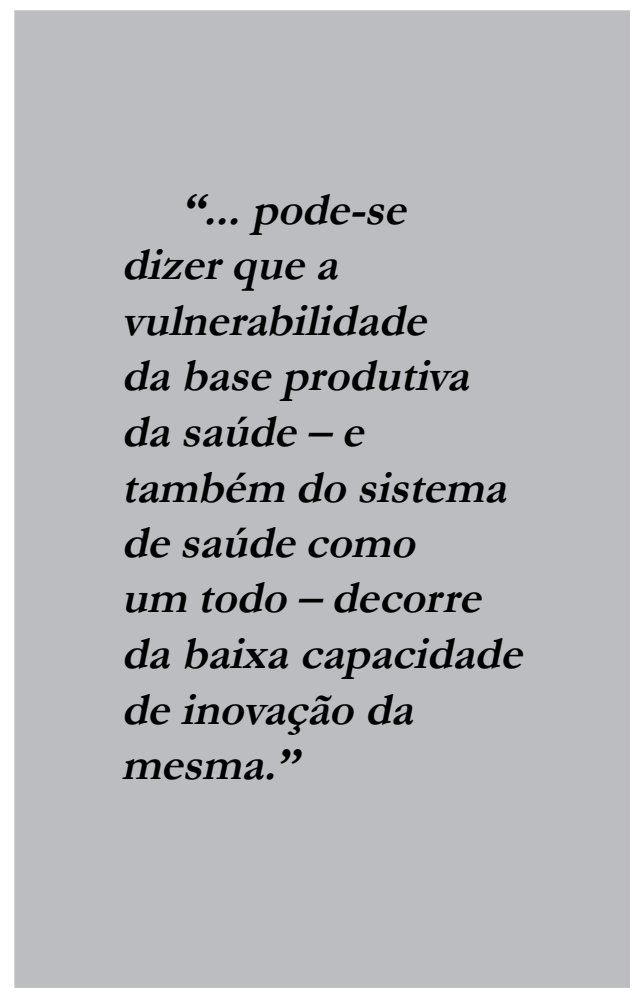

Ainda sobre as estratégias das empresas líderes, é importante observar que em seus processos de internacionalização concentram as atividades de maior densidade tecnológica e só descentralizam, aos países da periferia do sistema internacional, aquelas de menor valor agregado. Nesses países, observa-se uma dissociação entre as necessidades locais e os esforços privados 
em P\&D. Essa dinâmica é ressentida, especialmente pela insuficiência - e, em muitos casos, pela ausência - de pesquisas direcionadas aos principais agravantes da saúde, a exemplo de doenças tropicais (MALDONADO, 2012; Gadelha; Maldonado; Costa, 2012).

A respeito da concentração desse mercado e dos prejuízos sofridos pelos países que não dominam essas tecnologias da base produtiva da saúde, Oliveira, Labra e Bremudez (2006) destacam que as 100 maiores empresas farmacêuticas são responsáveis por $90 \%$ da produção mundial, sendo que $75 \%$ é consumida nos EUA, Japão e União Europeia. Nos últimos 10 anos, nenhuma das vinte empresas de maior faturamento bruto mundial lançou um único medicamento para qualquer uma das doenças negligenciadas (MÉDICOs SEM Fronteira, 2001).

\section{A indústria de base mecânica, eletrônica e de materiais da saúde}

O subsistema de base mecânica, eletrônica e de materiais envolve as indústrias de equipamentos e instrumentos mecânicos e eletrônicos, órteses e próteses e materiais de consumo em geral. Suas atividades são fortemente associadas às práticas médicas, determinando muitas vezes a tecnologia incorporada nos procedimentos adotados, no que se refere à prevenção, diagnóstico e tratamento de doenças (GADELHA; Maldonado; Costa, 2012; Maldonado et al., 2013).

Vale destacar, nesse subsistema, o papel da indústria de equipamentos - tanto pelo seu potencial de inovação, ao incorporar os avanços associados ao paradigma microeletrônico, quanto pelo seu impacto nos serviços, - que representa, de acordo com Gadelha, Maldonado e Costa (2012), uma fonte constante de mudanças nas práticas assistenciais e que, por sua vez, municia a tensão permanente entre a lógica da indústria (econômica) e a sanitária (social).

A exemplo do subsistema de base química e biotecnológica, o presente subsistema também se caracteriza como um oligopólio ${ }^{6}$ baseado na diferenciação de seus produtos, bastante especializados e com constantes atualizações tecnológicas em espaço de tempo relativamente curto (LEÃO; Oliveira; Albornoz, 2008). Essa atualização pressiona sobremaneira os custos da atenção à saúde, em especial porque as indústrias desse subsistema geralmente competem por meio do lançamento de equipamentos mais modernos e sofisticados.

Uma importante diferenciação do subsistema de base mecânica, eletrônica e de materiais, em relação ao previamente estudado, refere-se ao fato de que ele agrega à sua estrutura outros segmentos bastante diversificados; envolve desde bens de capital de alta complexidade (como diagnóstico por imagem), até materiais de consumo de uso rotineiro, passando por instrumentos, material cirúrgico e ambulatorial, seringas, entre outros.

De acordo com Maldonado et al. (2012), essa heterogeneidade, ao contrário do segmento farmacêutico, permite que empresas de menor porte oriundas de países em desenvolvimento ocupem posições relativamente importantes, abrindo-se, assim, nichos de mercado para a indústria local. No caso brasileiro, o subsistema de base mecânica, eletrônica e de materiais beneficia-se de uma base produtiva razoavelmente bem estruturada, fruto da política de substituição de importações vigente no período entre 1950 e 1980. Apesar de essa indústria ter sido bastante prejudicada pela abertura comercial da década de $1990^{7}$, o recente movimento de ampliação de acesso aos serviços de saúde dinamizou a demanda por equipamentos e materiais hospitalares 
e odontológicos, levando ao crescimento de $114,3 \%$ das vendas reais entre 1999 e 2009 (MALDONADO et al., 2012, p. 32).

\section{Serviços de saúde}

O subsistema de serviços é aquele de maior peso econômico do complexo da saúde, uma vez que responde por $64 \%$ da geração de bens e serviços de saúde no País ${ }^{8}$ (IBGE, 2012; Brasil, 2008). Sua importância é enfatizada tanto por ser estruturante do sistema de bem-estar quanto por seu papel na atividade econômica e no sistema nacional de inovação, uma vez que também articula novas tecnologias que emergem no esteio da $3^{\text {a }}$ Revolução Industrial, designadas de tecnologias portadoras de futuro, e que se referem a atividades produtivas intensivas em C\&T. Essas características dos serviços de saúde reforçam a ideia de que esse complexo produtivo possui potencial tanto para adensar o tecido produtivo quanto para direcioná-lo, de modo a compatibilizar a estrutura de oferta com a demanda social de saúde (GADELHA, 2007).

Vale notar que os serviços dinamizam a relação estabelecida com os demais subsistemas de base industrial do CEIS, estabelecendo o caráter sistêmico do mesmo, em especial por sua função de consumidor e demandante, muitas vezes influenciando e sendo influenciado pela produção de equipamentos médico-hospitalares, produtos farmacêuticos, imunoderivados, soros e demais insumos (Albuquerque; Cassiolato, 2000; Costa et al., 2012). Logo, é na interação entre setores com objetivos às vezes não consonantes que se buscam soluções para atender aos desafios postos pela conjugação das novas características epidemiológicas e da necessidade de redução dos crescentes custos com cuidados de saúde (Costa et al., 2012).
Note-se que não é trivial acomodar os interesses públicos e privados situados nessa agenda, não somente por sua importância social e econômica, como também por seu caráter estratégico e pelo porte e assimetria dos interesses envolvidos. Vale enfatizar que são vários os interesses em jogo quando se pensa em estruturar um sistema de saúde. Lassey et al. (1997 apud IBAÑEZ, 2011) chamam atenção, em especial, para os interesses referentes ao acesso, relevância econômica, características e tamanho do mercado industrial, pressão de custos, incorporação tecnológica, composição do financiamento e alterações das características epidemiológicas e demográficas.

Para se ter a dimensão desse mercado, Santos e Passos (2010) estimam que os gastos globais em serviços de saúde somaram US\$ 4 trilhões em 2007. No caso brasileiro, de acordo com o IBGE (2012), os serviços de saúde representam uma demanda de aproximadamente $\mathrm{R} \$ 250$ bilhões - se consideramos a manutenção da participação dos serviços observada no PIB de 2009, de 5,6\%, para o ano de 2012.

Assim, além das questões mais obviamente relacionadas à dinâmica desse subsistema (acesso e perfil sanitário), todo o arcabouço político-institucional da saúde, os interesses econômicos envolvidos, as tecnologias articuladas pelas indústrias do complexo da saúde, e os condicionantes da geração de conhecimento influenciam sobremaneira a dinâmica dos serviços de saúde. Nesse sentido, cabe refletir sobre o papel da Agência Nacional de Saúde Suplementar (ANS) no que tange à regulação dos planos de saúde, uma vez que os mesmos impactam sobremaneira o padrão de consumo e, logo, as tecnologias incorporadas no sistema nacional de saúde. 
Justamente porque lidam com segmentos tão dinâmicos da economia, os serviços de saúde têm-se organizado crescentemente como uma indústria, em bases empresariais de grande escala, tendo na capacidade de inovação o principal fator de dinamismo, competitividade e autonomia. Isso reafirma a importância de uma abordagem sistêmica e de seguir adensando o conhecimento desse subsistema (GADElHa et al., 2012), sob risco de os interesses privados manterem-se soberanos em relação aos sociais também no subsistema de serviços de saúde.

\section{O desafio da inovação no com- plexo produtivo da saúde}

Nos últimos anos, vem-se observando no Brasil a expansão do acesso aos serviços de saúde concomitante a mudanças nas características epidemiológicas, resultante do modelo político institucional do SUS, da evolução das condições socioeconômicas da população, da transição demográfica em curso e da veloz incorporação tecnológica por parte dos serviços de saúde. Como consequência, observa-se, entre outras coisas, um expressivo aumento da deman$\mathrm{da}$ por insumos relacionados à prestação e cuidados da saúde que, em contrapartida, ocasionou o crescimento das importações de insumos e produtos de saúde, refletindo uma situação de dependência, insustentável no médio e longo prazo.

Nesse contexto, tornou-se evidente a fragilidade da base produtiva da saúde, observada no crescimento do déficit da balança comercial do CEIS, particularmente a partir dos anos 2000. Tal cenário representa uma dupla ameaça, dado que, por um lado, decorre da fragilidade produtiva nacional em segmentos tecnológicos portadores de futuro e, por outro, traduz-se em um limitante para o projeto de universalização do SUS. Aponta, dessa forma, para a necessidade de fortalecer a dinâmica inovativa do CEIS, de maneira a desenvolver a produção nacional de insumos de saúde.

Como reflexo dessas evidências, o caráter estratégico do CEIS tem sido reconhecido em distintas políticas públicas a partir dos anos 2000. A política industrial e tecnológica e de comércio exterior (PITCE), lançada em 2003, foi pioneira nesse aspecto, ao reconhecer o potencial da cadeia produtiva farmacêutica para alavancar a incorporação de tecnologias portadoras de futuro, a exemplo da nanotecnologia, biotecnologia e química fina, entre outras (BRAsIL, 2003). Em 2007, no âmbito do Programa Mais Saúde, foi reconhecida a necessidade de fortalecimento da base produtiva do CEIS para reduzir a dependência externa em relação aos insumos críticos para a saúde e, portanto, a vulnerabilidade da política de saúde brasileira (BRAsIL, 2007).

Por sua vez, em 2008, a política de desenvolvimento produtivo (PDP) priorizou o complexo da saúde ao estimular a transferência de conhecimento científicotecnológico para a redução da vulnerabilidade do sistema nacional de saúde (BRAsIL, 2008). Em 2011, o Plano Brasil Maior destacou o complexo produtivo da saúde como uma das prioridades estratégicas para o desenvolvimento nacional. Por fim, em 2012, a Estratégia Nacional de Ciência, Tecnologia e Inovação enfatizou a necessidade de promoção de mecanismos de estímulo à inovação em saúde.

No entanto, apesar da crescente institucionalização da importância da saúde, a fragilidade do CEIS permanece evidente, conforme se verifica pela evolução do déficit de sua balança comercial (Gráfico 1). 


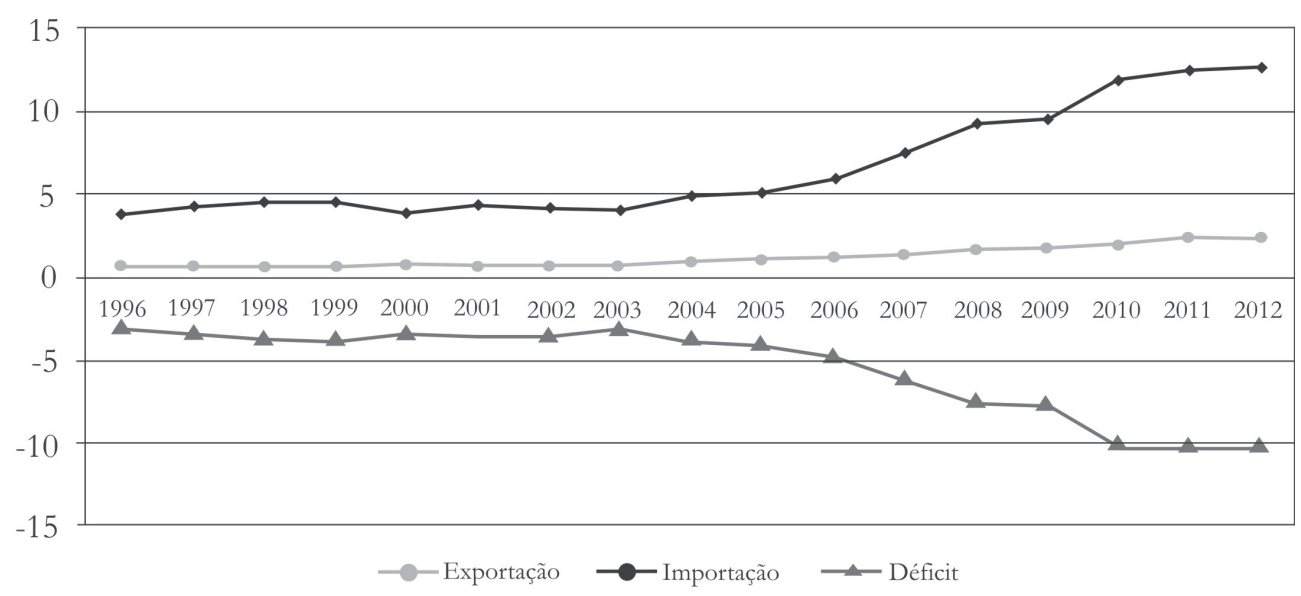

Fonte: Elaborado por GIS/ENSP/FIOCRUZ, a partir de dados da Rede Alice / MDIC. Acesso em janeiro/ 2013.

\section{Gráfico 1: Evolução da Balança Comercial da Saúde - CEIS 2012 (Valor em US\$ bilhões, atualizado pelo IPC/EUA)}

Ressalte-se que esse déficit saiu de um patamar de US\$ 3 bilhões em 2003, para ultrapassar US\$10 bilhões em 2012, revelando a vulnerabilidade do sistema de saúde no Brasil e da inserção competitiva internacional.

No que se refere à participação dos segmentos produtivos no déficit da balança comercial da saúde em 2012 (Gráfico 2), o subsistema de base química e de biotecnologia representa um saldo negativo de cerca de US\$ 8 bilhões ${ }^{9}$. Desse total, US\$ 2,8 bilhões são decorrentes do déficit com a importação de medicamentos, US\$2,4 bilhões com a importação de insumos farmoquímicos e US\$ 1,8 bilhão com a importação de hemoderivados. O restante refere-se à aquisição externa de vacinas, de reagentes para diagnóstico e de soros e toxinas, que totaliza cerca de US\$ 1 bilhão.

Conforme se observa no Gráfico 2, o déficit gerado pela importação de fármacos e medicamentos foi responsável por cerca de metade de todo o resultado negativo do balanço comercial do CEIS. De tal modo que, atualmente, esse setor revela particular vulnerabilidade da saúde, representando risco implícito para a implementação de políticas universais e integrais de acesso aos bens e serviços de saúde.

Vale notar que essa debilidade, observada nas indústrias do subsistema de base química e biotecnologia, é igualmente percebida nas indústrias do subsistema de base mecânica, eletrônica e de materiais, especialmente naqueles segmentos de maior complexidade tecnológica, cujo déficit atingiu, em 2012, o montante de US\$ 2,2 bilhões (Gráfico 2).

A despeito da baixa competitividade, é importante mencionar que esse subsistema - que reúne um conjunto particular de atividades com grande heterogeneidade tecnológica - oportuniza a existência de nichos competitivos em que o Estado pode atuar, no sentido de incentivar o fortalecimento da indústria nacional (Gadelha; Maldonado; Costa, 2012). 


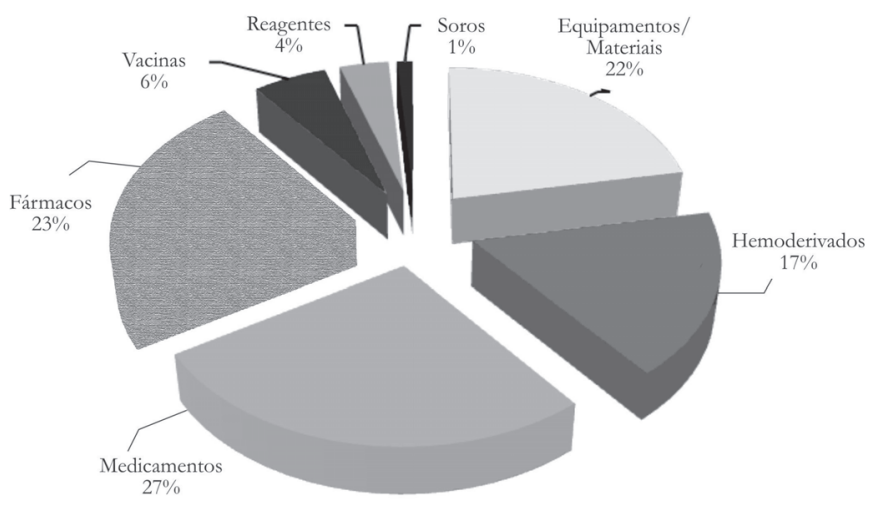

Fonte: Elaborado por GIS/ENSP/FIOCRUZ, a partir de dados da Rede Alice/MDIC. Acesso em janeiro/ 2013.

\section{Gráfico 2: Participação dos Segmentos do CEIS no Déficit da Balança Comercial da Saúde - 2012}

Quanto aos serviços de saúde, convém pontuar que os Gráficos 1 e 2 refletem, ainda que não completamente, a demanda pelos insumos, produtos e bens que são utilizados nesse subsistema. Ademais, a transição demográfica em curso e as novas características epidemiológicas ${ }^{10}$ implicarão tanto o aumento quanto a transformação das condições de demanda. Consequentemente, observar-se-á grande pressão sobre o sistema industrial por novas vacinas, medicamentos, equipamentos e, sobretudo, sobre a produção de serviços hospitalares, ambulatoriais e de diagnóstico, representando custos que o sistema de saúde, já subfinanciado ${ }^{11}$, não tem como suportar.

Nesse sentido, no que tange às fragilidades do CEIS, a principal questão a ser enfrentada é como estimular o desenvolvimento de produtos com alto valor social, de modo a reverter o quadro de vulnerabilidade ao qual o sistema de saúde encontra-se exposto, tendo em vista, inclusive, a inserção competitiva internacional. Vale destacar que, no Brasil, se observa uma baixa competitividade do CEIS em face do mercado mundial, em função de fatores diversos atinentes tanto ao próprio modelo e estrutura do Estado ${ }^{12}$ quanto a questões mais setoriais.

O grande dilema que se apresenta ao complexo é que o contexto nacional se caracteriza por uma dupla desarticulação. Por um lado, em que pesem todas as iniciativas governamentais recentes em relação à saúde, esta ainda não é abordada de forma sistêmica na agenda de desenvolvimento nacional. Por outro, a despeito da base científica instalada no País e da existência de base industrial diversificada, esta não é inovativa, uma vez que a natureza e intensidade das interações estabelecidas entre ambas não têm propiciado processos inovativos. Observa-se, dessa forma, o afastamento da empresa em relação à base científica do País, decorrente de características e desarticulações do sistema nacional de inovação, que está marcado por uma baixa capacidade inovativa. Por esses 
motivos, no Brasil, a base produtiva da saúde é o elo fraco do sistema nacional de inovação em saúde.

Além disso, Chaves e Albuquerque (2006) apontam a existência de um "limiar de produção científica" necessário para iniciar circuitos virtuosos entre ciência e tecnologia, situado em 150 artigos por milhão de habitantes. De acordo com os autores, em meados dos anos 2000, o Brasil alcançava a marca de 63 artigos por milhão de habitantes, portanto abaixo do citado limiar. Cabe lembrar que a produção bibliográfica científica não basta por si só para gerar inovações, uma vez que essa precisa ser articulada com a base industrial.

Em função dessas evidências, pode-se dizer que a vulnerabilidade da base produtiva da saúde - e também do sistema de saúde como um todo - decorre da baixa capacidade de inovação da mesma.

Cabe ressaltar, ademais, que características do arcabouço institucional também não têm incentivado que as inovações se orientem socialmente. A pertinência dessa questão encontra eco em Costa e Gadelha (2012) e em Viana, Silva e Elias (2007), na medida em que se percebe que a incorporação tecnológica no Brasil enfrenta forças assimétricas quanto aos interesses sanitários e econômicos, responsáveis pelo estabelecimento de uma relação hierárquica mais favorável aos interesses econômicos (VIDOTTI; Castro; Calil, 2008).

Visando a aprofundar o entendimento sobre processos inovativos da saúde, temse utilizado o arcabouço teórico do sistema nacional de inovação em saúde (SNIS). A partir de estudos seminais elaborados por Freeman (1987), Lundvall (1988) e Nelson (1993), autores diversos, a exemplo de Albuquerque e Cassiolato (2002), vêm propondo a aplicação do conceito de sistema nacional de inovação (SNI) ao setor da saúde ${ }^{13}$. Dentro dessa corrente teórica, a geração, uso e difusão de inovações são percebidos como respostas aos "processos sistêmicos que permitem às empresas e demais organizações aprender, usar e acumular capacitações e desenvolver novos produtos e processos" (Lastres; Cassiolato, 2007, p. 153). No caso do SNIS, essas interações envolvem atores diversos: universidades e instituições de pesquisa; hospitais, clínicas e postos de saúde; instituição de regulação (Agência Nacional de Vigilância Sanitária); indústria farmacêutica, de equipamentos e materiais hospitalares; e órgãos públicos voltados para a área da saúde pública. A Figura 2 apresenta o conjunto dos atores envolvidos e suas interações no caso de SNIS maduro.

Ressalta-se que, pelo fato de o SNIS envolver uma forte institucionalidade público-privada, surge uma janela de oportunidade para potencializar o processo de geração de inovação em saúde, dado o marcante protagonismo do Estado - que, a um só tempo, se configura como importante demandante e comprador de bens e serviços, como também responde pelo aparato institucional desse sistema, além de outras questões relacionadas a investimento, prestação de serviços de saúde etc.

Assim, numerosas iniciativas foram empreendidas visando ao fortalecimento do CEIS, que se configura como o elo frágil do SNIS, por meio da intensificação das interações entre atores públicos e privados e de medidas de fortalecimento das indústrias nacionais relacionadas ao CEIS, a exemplo da transferência de tecnologia entre empresas farmoquímicas e laboratórios oficiais, e programas para o fortalecimento $\mathrm{da}$ base produtiva e inovativa nacional. 


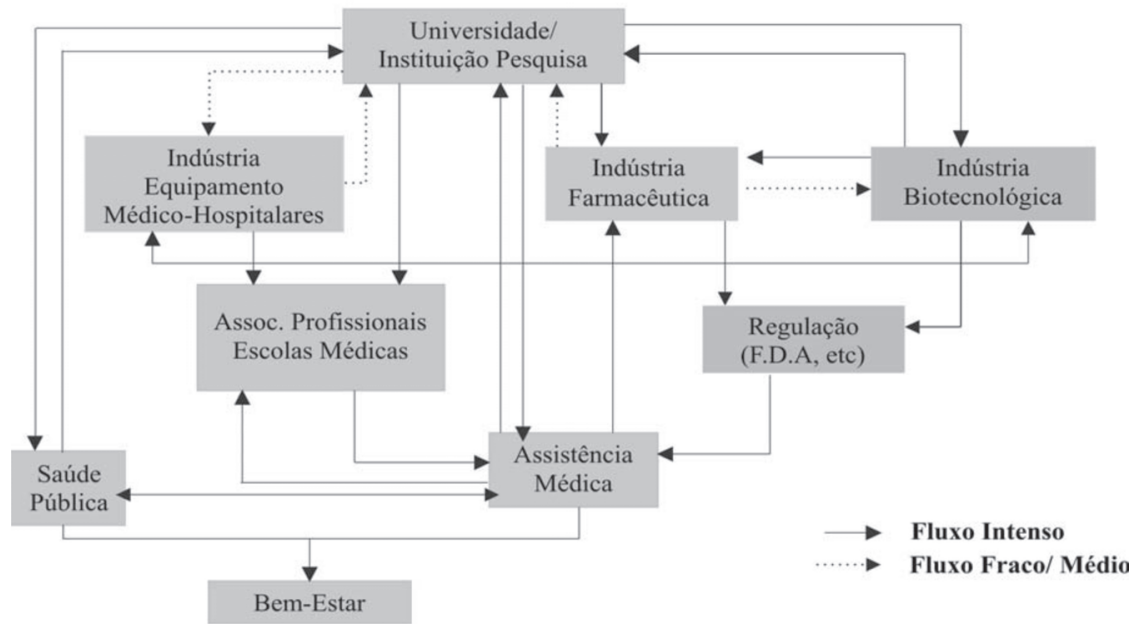

Fonte: Chaves e Albuquerque (2006, p. 525).

Figura 2: Fluxos de informações científicas e tecnológicas no sistema de inovação do setor saúde: o caso de países com sistemas maduros

Vale ressaltar uma particularidade nacional, referente à existência de uma rede de laboratórios oficiais capazes de potencializar a atuação pública e a orientação social do desenvolvimento tecnológico nacional. Um exemplo emblemático refere-se à experiência pioneira da produção do Efavirenz pelo laboratório público Farmanguinhos da Fiocruz (em parceria com três empresas farmoquímicas nacionais), que serviu de base para que o Ministério da Saúde institucionalizasse, em 2009, uma política de desenvolvimento produtivo, viabilizando parcerias similares com um marco legal mais estável. Destaca-se, ainda, a consolidação da rede nacional de laboratórios públicos com a construção da Hemobrás em Pernambuco, do novo Instituto Nacional de Traumatologia e Ortopedia (INTO) e do novo Campus Integrado do Instituto Nacional do Câncer (INCA) no Rio de Janeiro, evidenciando a mobilização do Estado no campo da inovação em saúde.

Por fim, as vultosas compras públicas de insumos de saúde para o funcionamento do SUS também têm potencial para insuflar a dinâmica inovativa do CEIS, por meio do uso do poder de compra do Estado ${ }^{14}$.

No entanto, apesar dessas importantes iniciativas, ainda não se alcançou um nível satisfatório de interação entre os atores envolvidos, notadamente entre indústria e institutos de pesquisa/universidades no SNIS, de maneira a fortalecer a dinâmica inovativa do CEIS, como evidenciado pelo crescente déficit de sua balança comercial.

Adicionalmente, Costa et al. (2012) chamam atenção para limitações vivenciadas por instituições de regulação, a exemplo da ANVISA ${ }^{15}$, assim como para a necessidade de aprimoramento do marco regulatório no que se refere às políticas voltadas para o desenvolvimento produtivo (uso do poder de compra do Estado, transferência tecnológica, margem de preferência). Nessa mesma linha, sugerese a revisão do papel da ANS no que se refere à mediação dos interesses públicoprivados vigentes na agenda da saúde, no sentido de incentivar um padrão de 
consumo em saúde que seja socialmente inclusivo.

Nesse contexto, o complexo mostrase pouco articulado tanto com relação à base de conhecimento nacional - reconhecidamente forte na área da saúde - quanto para o desenvolvimento de um sistema nacional equânime e universal em saúde. $\mathrm{O}$ que suscita, assim, a necessidade não somente de se definir prioridades no fomento à competitividade das indústrias nacionais de saúde (que muito avançou nos últimos anos), como de se qualificar e intensificar as políticas e mecanismos de fomento à capacidade produtiva e de inovação do CEIS. A ideia é conduzi-lo para patamares de maior densidade tecnológica e buscar uma produção que seja orientada socialmente, retomando o conceito de Furtado, para quem "desenvolvimento" relaciona-se tanto com homogeneização social quanto com o desenvolvimento de um sistema produtivo eficiente que apresente uma relativa autonomia tecnológica (FurTAdo, 1964).

Advoga-se, nesse sentido, pela superação da histórica polaridade modernização-marginalização nacional (FURTADO, 1964; Albuquerque, 2007; Saboia; Carvalho, 2007). De forma análoga, caso o complexo da saúde não seja abordado de forma sistêmica e não se mediem os interesses sociais e econômicos, corre-se o risco de se observar um crescente distanciamento das indústrias da saúde em relação aos princípios do SUS.

\section{Considerações finais}

A pertinência da utilização do arcabouço teórico da economia política e do instrumental dos sistemas de inovação na análise é evidenciada quando se busca um olhar sistêmico da saúde, que inclui a sua vertente social, mas não se limita a essa. O CEIS, ao relacionar também a dimensão econômica da saúde, revela seu caráter sistêmico, apresentando potencial para a superação da dicotomia observada entre a lógica econômica e a sanitária.

Uma articulação virtuosa entre essas duas dimensões, em especial considerandose o caráter estratégico das tecnologias por ela relacionadas, pode orientar um padrão de inovação tecnológica, público e privado, que aproxime a produção das necessidades locais e que, adicionalmente, permita um salto qualitativo em um ambiente internacional extremamente competitivo.

No entanto, na atualidade o CEIS enfrenta um duplo desafio. Por um lado, apesar de ter sido objeto de uma série de programas de políticas públicas nos últimos anos, a saúde ainda não é abordada integralmente de forma sistêmica na agenda de desenvolvimento nacional. Por outro, sua base produtiva ainda não apresenta uma dinâmica inovativa capaz de torná-la competitiva em nível internacional, como se evidencia ao analisar a evolução da balança comercial do CEIS. Cabe destacar que esses desafios não são exclusivamente relacionados às indústrias desse complexo, mas condicionam a capacidade do País para universalizar de fato seu sistema de saúde.

Para reverter esse quadro, é necessário adensar o conhecimento acerca da dinâmica inovativa da base produtiva da saúde. Nesse sentido, o arcabouço teórico do SNIS constitui uma ferramenta conceitual rica em perspectivas para nortear uma agenda futura de pesquisa, com o intuito de formular políticas públicas capazes de orientar socialmente o desenvolvimento tecnológico e produtivo da saúde. A intensificação e qualificação do uso do poder de compra do Estado, das parcerias para o desenvolvimento produtivo, além 
do fortalecimento da rede de laboratórios públicos e o aprimoramento do marco regulatório relativo ao fortalecimento do CEIS constituem opções promissoras para alcançar tamanho objetivo.

A partir do exposto no artigo, sugerese que se intensifique o uso de tais instrumentos de ação pública, de maneira a conferir autonomia à base produtiva da saúde, tornando, dessa maneira, sustentável o projeto de universalização do Sistema Único de Saúde, conforme estabelecido na Constituição de 1988.

(Artigo recebido em janeiro de 2013. Versão final em junho de 2013).

\section{Notas}

${ }^{1}$ Neste artigo, as terminologias "complexo da saúde", "complexo produtivo" ou "complexo econômico-industrial da saúde" (CEIS) serão utilizadas como sinônimos ao se referirem ao conjunto de segmentos produtivos (industriais e de serviços) que estabelecem uma relação sistêmica entre si, envolvidos na prestação de serviços de saúde.

${ }^{2}$ A retração do Estado e a falta de política industrial no período afetam todo o parque produtivo brasileiro. Além disso, dado a saúde ser intensiva em tecnologias portadoras de futuro, os efeitos deletérios da abrupta abertura comercial dos anos 1990 foram de grande alcance, também no que diz respeito à competitividade internacional da economia brasileira.

${ }^{3}$ De acordo com a prospecção do mercado farmacêutico desenvolvida pelo IMS Health (2012), o gasto total dos países emergentes deve quase dobrar no período 2011-2016, passando de US\$ 194 bilhões em 2011 para US\$ 359 bilhões em 2016 (no mesmo período, o mercado brasileiro deve dar um salto de US\$ 30 bilhões para US\$ 55 bilhões).

${ }^{4}$ Dados extraídos da apresentação institucional da Secretaria de Ciência, Tecnologia e Insumos Estratégicos do Ministério da Saúde (2009).

${ }^{5}$ Disponível em: www.Pharmexec/article/article. Acessado em: 20/12/2011.

${ }^{6} \mathrm{~A}$ concentração deste mercado é tamanha, que as vinte maiores empresas de equipamentos e materiais médico-hospitalares respondem por cerca de $70 \%$ da produção em nível mundial (The World Medical Markets Facts Book, 2010).

${ }^{7}$ De acordo com Maldonado (2012, p. 6), "a década de 1990 foi marcada por transformações estruturais no funcionamento desta indústria [de equipamentos e materiais hospitalares] em decorrência do processo de abertura comercial, o que significou uma crescente dependência do País em relação às importações de equipamentos, sobretudo de maior densidade tecnológica".

${ }^{8}$ Segundo dados do IBGE (2012), a saúde respondeu por 8,8\% do PIB em 2009; desses, $5,6 \%$ referem-se somente aos serviços.

${ }^{9}$ Dados elaborados por GIS/ENSP/FIOCRUZ, 2012, a partir de dados da Rede Alice/ MDIC. Acesso em: janeiro/2012.

${ }^{10}$ As características epidemiológicas da população apontam para significativas transformações no que tange ao aumento de doenças crônicas.

${ }^{11} \mathrm{O}$ subfinanciamento da saúde, em especial, no que se refere à parcela de origem pública, é incompatível em um país como o Brasil, onde $76 \%$ da população depende exclusivamente do 
Sistema Único de Saúde e a participação pública nos gastos sanitários gira em torno de $46 \%$ (GAdelHa; Costa, 2012). Este, inclusive, representa um dos principais desafios setoriais que precisam ser vencidos para a superação da fragilidade do SUS.

${ }^{12}$ A exemplo das características do sistema tributário nacional, da infraestrutura instalada, do sistema educacional e sua consequências para a formação das competências nacionais, entre outros.

${ }^{13}$ Outros autores já tinham delineado pistas teóricas nesse sentido, a exemplo de Hicks e Katz (1996), ao apontar a existência de um sistema biomédico de inovação, e de Gelinjs e Roseberg (1995), ao estudar interações entre universidades e indústrias na geração de inovações médicas.

${ }^{14}$ A Lei no 12.349/2010, regulamentada pelo Decreto ํํ⒎546/2011, que prevê margem de preferência de até $25 \%$ para a compra a produtores nacionais (sendo possível considerar no cálculo geração de emprego e renda, impacto na arrecadação de impostos, desenvolvimento nacional), constitui um instrumento importante para materializar esse potencial.

${ }^{15} \mathrm{O}$ prazo médio de aprovação de um remédio por parte da Anvisa, afetada por uma estrutura de recursos humanos subdimensionada, chegou, no final de 2012, a 640 dias, contra um máximo de 180 dias para produtos considerados como estratégicos nos Estados Unidos (O Estado de São Paulo, 26/11/2012).

\section{Referências bibliográficas}

Albuquerque, E. M. \& Cassiolato, J. E. As especificidades do sistema de inovação do setor saúde. Revista de Economia Política, v. 22, n. 4(88), p. 134-151, 2002.

- As especificidades do sistema de inovação do setor saúde: uma resenha da literatura como introdução a uma discussão sobre o caso brasileiro. Belo Horizonte: Fesbe; 2000. Albuquerque, E. M. Celso furtado, a polaridade modernização-marginalização e uma agenda para a construção dos sistemas de inovação e de bem-estar social. In: SABOIA, J. e Carvalho, F. J. C. (Org). Celso Furtado e o século XXI. Barueri, SP: Manole; Rio de Janeiro: Instituto de Economia da Universidade Federal do Rio de Janeiro, 2007. p. 181 - 203.

Angell, M. A verdade sobre os laboratórios farmacêuticos. Rio de Janeiro: Record, 2007.

Brasil. Mais saúde: direito de todos: 2008 - 2011. Ministério da Saúde, Secretaria-Executiva. $2^{\text {a }}$ ed. Brasília, DF: Editora do Ministério da Saúde, 2008. Disponível em: <http://bvsms.saude.gov.br/bvs/publicacoes/mais_saude_direito_todos_2ed.pdf $>$. Acesso em: julho de 2011.

Brasil. Ministério da Saúde. Programa Mais Saúde: direito de todos - 2008-2011 (PAC Saúde). $1^{\mathfrak{a}}$ ed. Brasília/DF: Editora do Ministério da Saúde, 2007.

Brasil. Ministério do Desenvolvimento, Indústria e Comércio Exterior. Política Industrial, Tecnológica e de Comércio Exterior. 2003. Página web: http://www.camara-e.net/_upload/ 20031126Diretrizes.pdf.

Politica de Desenvolvimento Produtivo (PDP). 2008. Página web: http:// www.desenvolvimento.gov.br/pdp/arquivos/destswf1224095287.ppt. 
Chaves, C. V.; Albuquerque, E. M. Desconexão no sistema de inovação no setor saúde: uma avaliação preliminar do caso brasileiro a partir de estatísticas de patentes e artigos. Economia Aplicada, 10 (4): 523-539, 2006.

Costa, L. S.; Gadelha, C. A. G. Análise do Subsistema de Serviços em Saúde na dinâmica do Complexo Econômico-Industrial da Saúde. In: Fundação Oswaldo Cruz. $A$ saúde no Brasil em 2030: diretrizes para a prospecção estratégica do sistema de saúde brasileiro. Rio de Janeiro, 2012. No prelo.

Costa, L. S. et al. Dinâmica Inovativa para a Reestruturação dos Serviços de Saúde. Revista de Saúde Pública da USP, Suplemento Especial Saúde e Desenvolvimento, São Paulo, v.46, p. 76-82, dez. 2012.

Faveret, Filho; P \& Oliveira, P. J. A. A universalização excludente: reflexões sobre as tendências do sistema de saúde. Planejamento e Políticas Públicas, 3: 139-82, 1990.

FrEEMAn, C. Technology policy and economic performance: lessons from Japan. London: Pinter; 1987. Technology Policy and Economic Performance: Lessons from Japan. Pinter, London, 1987.

Furtado, C. Dialética do Desenvolvimento. Rio de Janeiro: Ed. Fundo de Cultura, 1964. . O capitalismo global. Rio de Janeiro: Ed. Paz e Terra, 1998.

Gadelha, C. A. G. O complexo industrial da saúde e a necessidade de um enfoque dinâmico na economia da saúde. Ciência e Saúde Coletiva, 8(2):521-35, 2003. DOI:10.1590/ S1413-81232003000200015

. Desenvolvimento e Saúde: em busca de uma nova utopia. Revista Saúde em Debate, Rio de Janeiro, v. 19, n. 71, p. 326-327, set/dez. 2007.

. Desenvolvimento, Complexo Industrial da Saúde e Política Industrial. Revista de Saúde Pública/Journal of Public Health, São Paulo, v. 40, n. Especial, p. 11-23, 2006.

Estudo da competitividade de cadeias integradas no Brasil: impactos das zonas livres de comércio (Cadeia: Complexo da Saúde). Campinas: IE/Neit/Unicamp/MCTFINEP/MDIC, (Nota Técnica Final). 2002.

Gadelha, C. A. G. \& Costa, L. S. Saúde e Desenvolvimento no Brasil: estado da arte e desafios. Revista de Saúde Pública da USP, Suplemento Especial Saúde e Desenvolvimento, São Paulo, 2013.

. Saúde e desenvolvimento Nacional: a gestão federal entre 2003 e 2010. In: Machado, C. V.; Baptista, T. W. F.; Lima, L. D. (Org). Política de saúde no Brasil: continuidades e mudanças. Rio de Janeiro: Fiocruz, 2012.

Gadelha, C. A. G.; Maldonado, J. M. S. V.; Costa, L. S. O complexo produtivo da saúde e sua relação com o desenvolvimento: um olhar sobre a dinâmica da inovação em saúde. In: Giovanella, L; Escorel, S; Lobato, L. V. C.; Noronha, J. C.; Carvalho, A. I. Organizadores. Políticas e sistemas de Saúde no Brasil. $2^{a}$ edição revista e ampliada. Rio de Janeiro: Editora Fiocruz; 2012: p. 209-237.

Gadelha, C. A. G. et al. A dinâmica do sistema produtivo da saúde: inovação e complexo econômico-industrial. Rio de Janeiro: Ed. Fiocruz, 2012. 221p. 
Gadelha, C. A. G.; Quental, C \& Fialho, B. C. Saúde e Inovação: uma abordagem sistêmica das indústrias da saúde. Cadernos de Saúde Pública, Rio de Janeiro, 19 (1), p. 47-59, jan.-fev. 2003.

Global Booz \& Company. Global Innovation 1000. 2012. Disponível em: http:// www.booz.com/media/uploads/BoozCo_The-2012-Global-Innovation-1000-Study.pdf Guimarães, R. Pesquisa em saúde no Brasil: contexto e desafios. Revista de Saúde Pública, 40 (N Esp): 3-10, 2006.

Ibañez, N . Análise comparada de Sistemas de Saúde. In: Ibañez, N; Elias, PEM; Seixas, PHD (org). Política e Gestão Pública em Saúde. São Paulo: Hucitec: Cealag, 2011.

IMS Institute for Healthcare Informatics . The Global Use of Medicines: Outlook through 2016. 2012. Disponível em: http://www.imshealth.com/deployedfiles/ims/Global/ Content/Insights/IMS\%20Institute \%20for $\% 20$ Healthcare $\% 20$ Informatics / Global\%20Use\%20of\%20Meds\%202011/Medicines_Outlook_Through_ 2016_Report.pdf

Instituto Brasileiro de Geografia e Estatística. Conta satélite da saúde: diretoria de pesquisas, mordenação de contas nacionais, sistema de contas nacionais (2007-2009). Rio de Janeiro, 2012.

Lastres, H.. M. M. e Cassiolato, J. E. Inovação e sistemas de inovação: relevância para a área de saúde. RECIIS -Revista Eletrônica de Comunicação, Informação \& Inovação em Saúde . Rio de Janeiro, v.1, n.1, p.153-162, 2007.

LeÃo, R; Oliveira, E \& Albornoz, L. Estudo setorial - setor de equipamentos e materiais de uso em saúde. Secretaria de Ciência, Tecnologia e Insumos Estratégicos, Departamento de Economia da Saúde, Coordenação Geral de Economia da Saúde. Brasília, 2008.

Lundvalt, B. A. Innovation as an interactive process: from user-producer interaction to the national system of innovation. In: Dosi, G; Nelson, R; Silverberg, G; Soete, L (Ed.). Technical change and economic theory. London: Printer Publishers, 1988. p. 349-369.

Machado, C. V.; Lima, L. D. \& Baptista, T. W. F. Desenvolvimento, Sistema de proteção Social e Saúde: correntes teóricas e perspectiva histórica. Fase 1 do Projeto Desenvolvimento e Saúde: consolidação e disseminação do marco conceitual. Gadelha, C. A. G. (Coord.) e Maldonado, J. M. S. V (Coord. exec.). Grupo de Pesquisa sobre o Complexo Industrial e Inovação em Saúde, da ENSP. Rio de janeiro, setembro de 2008.

Maldonado, J. M. S. V. A inserção do CEIS na política de desenvolvimento nacional: perspectivas para 2022/2030. In: Fundação Oswaldo Cruz. A saúde no Brasil em 2030: diretrizes para a prospecção estratégica do sistema de saúde brasileiro. Rio de Janeiro, 2012. No prelo.

Maldonado, J.; Gadelha, C. A .G.; Vargas, M. A.; Costa, L. S. A Dinâmica Inovativa do Subsistema de Base Mecânica, Eletrônica e de Materiais. Revista de Saúde Pública da USP, Suplemento Especial Saúde e Desenvolvimento, São Paulo, 2012.

Maldonado, J. et al. Subsistema de Base Mecânica, Eletrônica e de Materiais do Complexo Econômico Industrial da Saúde: perspectivas para 2022/2030. In: Fundação Oswaldo Cruz. A saúde no Brasil em 2030: diretrizes para a prospecção estratégica do sistema de saúde brasileiro. Rio de Janeiro: Fiocruz, 2012. No prelo. 
Manfredini, M. A. Características da Indústria de Equipamentos Odontológicos e de Produtos para Higiene Bucal no Brasil entre 1990 e 2000. Dissertação de mestrado. Programa de Pós-Graduação em Ciências. São Paulo. 2006. 141p.

Médicos Sem Fronteiras. Desequilíbrio fatal: a crise em pesquisa e desenvolvimento de drogas para doenças negligenciadas. Grupo de Trabalho de Drogas para Doenças Negligenciadas, Médicos Sem Fronteiras, Geneva. 2001.

Mendes, E.V. Uma agenda para a saúde. São Paulo: Hucitec, 1996.

Nelson, R. R. National Innovation Systems: comparative analysis. Oxford University Press, New York. 1993.

Oliveira, E. A; Labra, M. E; Bremudez, J. (2006) A produção pública de medicamentos no Brasil: uma visão geral. Cadernos de Saúde Pública, v.22, n.11, Rio de Janeiro, 2006. DOI: 10.1590/S0102-311X2006001100012.

Paim, J. S. Bases Conceituais da Reforma Sanitária Brasileira. In: Fleury, S. (Org.). Saúde e democracia: A luta do CEBES. São Paulo: Lemos Editorial, 1997.

Rundvalt, D.; Albuquerque, E. S. Complexo Industrial-Militar-Farmacêutico e a construção da hegemonia norte-americana no séc. XXI. Revista de Geopolítica. 2012. Disponível em: http://www.revistageopolitica.com.br/ojs/ojs-2.2.3/index.php/rg/article/ viewArticle/63

Saboia, J. e Carvalho, F. J. C. Celso Furtado e o Século XXI. Barueri/SP: Manole; Rio de Janeiro: Instituto de Economia da UFRJ, 2007.

Santos, M. A. B. e Passos, S. R. L. Comércio internacional de serviços e complexo industrial da saúde: implicações para os sistemas nacionais de saúde. Cad. Saúde Pública, v. 26, $\mathrm{n}^{\circ}$ 8, Rio de Janeiro, 2010.

Soete, L. Verspagen, B. Weel, B. T. Systems of innovation. 2010. In: Bronwyn, H.H.; Rosenberg, N. Handbook of economics of innovation. Amsterdam: Elsevier, 2010. v. 2, p.11591180.

Temporão, J. G. O complexo industrial da saúde: público e privado na produção e consumo de vacinas no Brasil. 2002. Tese (Doutorado) - Universidade do Estado do Rio de Janeiro, Rio de Janeiro.

The World Medical Markets Fact Book. World Leaders in Health Industry Analysis. Espicom Business Intelligence, 2010. Disponível em: http://www.espicom.com/ web3.nsf/structure/tocmedrep10/\$file/wmmfb10_toc.pdf. Acesso em: outubro de 2012.

Tigre, P. B. Gestão da Inovação: a economia da tecnologia do Brasil. Rio de Janeiro: Elsevier, 2006. UgÁ, M. A. D. \& Marques, R. M.. O Financiamento do SUS: trajetória, contexto e constrangimentos. In: Lima, N. T.; Gerschman, S \& Edler, F. C. (Org). Saúde e Democracia: história e perspectivas do SUS. Rio de janeiro: Editora Fiocruz, 2005. p. 193-233.

VARGAS, M. A.et al. Reestruturação na indústria farmacêutica mundial e seus impactos na dinâmica produtiva e inovativa do setor farmacêutico brasileiro. In: XV ENCONTRO NACIONAL DE ECONOMIA POLÍTICA, 2010, São Luis do Maranhão. 
Viana, A. L.; Elias, P. E. M.. Saúde e desenvolvimento. Ciência e Saúde Coletiva. 2007;12 Supl:1765-77. DOI:10.1590/S1413-81232007000700002

Viana, A. L.; Nunes, A. A.; Silva, H. P. Complexo Produtivo da Saúde, desenvolvimento e incorporação de tecnologias. In: Inañez, N; Elias, PEM; e Seixas, PHd'A (Org). Política e Gestão Pública em Saúde. São Paulo: Hucitec: Cealag, 2011.

Viana, A. L.; Silva, H. P. \& Elias, P. E. M. Economia política da saúde: introduzindo o debate. Divulgação em Saúde para Debate, (37): 7-20, 2007.

Viana, A. L. \& Elias, P. E.. Saúde e desenvolvimento. Ciência e Saúde Coletiva, v. 12, Suplemento, p. 1765-1776, 2007.

Victora, C. G.; Barreto, M. L.; Leal, M. C; Monteiro, C. A.; Schmidt, M. I.; Paim, J.; Bastos, F. I.; Almeida, C.; Bahia, L.; Travassos, C.; Reichenheim, M.; Barros, F. C. Condições de saúde e inovações nas políticas de saúde no Brasil: o caminho a percorrer. 2011. Disponível em: www.thelancet.com Séries, DOI: 10.1016/S0140-6736(11)60055-X

Vidotti, C. C. F.; Castro, L. L. C. e Calil, S. S.. New drugs in Brazil: do they meet Brazilian public health needs? In: Rev Panam Salud Publica / Pan Am J Public Health 24 (1), p. 36-45, 2008.

Who (World Health Organization) . Global Health Observatory Data Repository. 2010. Disponível em: http://apps.who.int/ghodata/?theme=country Acesso em: abril de 2012. 


\section{Resumo - Resumen - Abstract}

\section{O complexo produtivo da saúde e sua articulação com o desenvolvimento socio-} econômico nacional

Laís Silveira Costa, Carlos Angusto Grabois Gadelha, José Maldonado, Marcelo Santo e Antoine Metten

O objetivo deste artigo é aprofundar o conhecimento sobre o complexo econômico-industrial da saúde (CEIS) e a dinâmica de seus subsistemas, identificando os principais desafios desse complexo no Brasil e as ameaças à manutenção de um sistema de saúde que se pretende universal. A relevância desse estudo situa-se no reconhecimento de que, não obstante a importância da saúde e de sua base produtiva na agenda de desenvolvimento nacional, sua fragilidade representa crescente vulnerabilidade para o sistema de saúde nacional, enfatizando, inclusive, a necessidade de adensar a base de conhecimento científico sobre o tema. Para a sua elaboração, adotou-se uma abordagem sistêmica, propiciada tanto pelo arcabouço da economia política quanto pelo instrumental teórico dos sistemas de inovação (SI).

Palavras-chave: saúde; complexo econômico-industrial da saúde; setor produtivo; desenvolvimento

\section{El complejo productivo de la salud y su articulación con el desarrollo socioeconómico nacional}

Laís Silveira Costa, Carlos Augusto Grabois Gadelha, José Maldonado, Marcelo Santo y Antoine Metten

El objetivo de este trabajo es profundizar en el conocimiento del complejo económicoindustrial de la salud (CEIS) y de la dinámica de sus subsistemas, identificando los principales desafíos de este complejo en Brasil y las amenazas para el mantenimiento de un sistema de salud que se pretende que sea universal. La importancia de este estudio radica en el reconocimiento de que, a pesar de la importancia de la salud y de su base de producción en la agenda nacional de desarrollo, su fragilidad representa la creciente vulnerabilidad del sistema nacional de salud, con énfasis en la necesidad de aumentar la base de conocimientos científicos sobre el tema. Para su elaboración, hemos adoptado un enfoque sistémico, impulsado tanto por el marco de la economía política como las herramientas teóricas de los sistemas de innovación (SI).

Palabras clave: salud; complejo económico-industrial de la salud; sector productivo; desarrollo

Health Economic-Industrial Complex and national economic and social development Laís Silveira Costa, Carlos Augusto Grabois Gadelha, José Maldonado, Marcelo Santo and Antoine Metten

The aim of this paper is to deepen the knowledge about the Health Economic-Industrial Complex (CEIS) and dynamics of its subsystems, identifying the main challenges of this complex in Brazil and threats to maintaining a health care system that is intended to be universal. The relevance of this study lies in the recognition that despite the importance of health and its production base in the national development agenda, its fragility means increased vulnerability for the national health system, emphasizing the need to increase the base of scientific knowledge on the subject. For its elaboration, we adopted a systemic approach, fostered by both the framework of political economy as the theoretical tools of innovation systems (IS).

Keywords: health; health economic-industrial complex; productive sector; development 


\section{Laís Silveira Costa}

É doutora em Saúde Pública pela Escola Nacional de Saúde Pública Sérgio Arouca da Fiocruz (ENSP/Fiocruz) e mestre em Development Studies pela London School of Economicis and Political Science. É Analista de Gestão da Inovação em Saúde da Fiocruz, coordenadora adjunta do Grupo de Pesquisa de Inovação em Saúde da Fiocruz e coordenadora científica do Boletim Informativo do Complexo Econômico-Industrial da Saúde. Contato: laiscosta@fiocruz.br

Carlos Augusto Grabois Gadelha

É doutor em Economia pelo Instituto de Economia da Universidade Federal do Rio de Janeiro (UFRJ). Tem atuado como Secretário de Ciência, Tecnologia e Insumos Estratégicos do Ministério da Saúde, coordenador do mestrado profissional em Política e Gestão de CT\&I em Saúde da ENSP/Fiocruz e coordenador do Grupo de Pesquisa de Inovação em Saúde da Fiocruz.Contato: carlos.gadelha@saude.gov.br

José Maldonado

É doutor em Engenharia de Produção pela Universidade Federal do Rio de Janeiro (UFRJ) e coordenador adjunto do mestrado profissional de Política e Gestão em CT\&I em Saúde da ENSP/Fiocruz e pesquisador do Grupo de Pesquisa de Inovação em Saúde da Fiocruz. Contato: jose.maldonado@ensp.fiocruz.br

\section{Marcelo Santo}

É doutorando em Saúde Pública pela Escola Nacional de Saúde Pública Sérgio Arouca da Fiocruz (ENSP/Fiocruz) e pesquisador do Grupo de Pesquisa de Inovação em Saúde da Fiocruz. Contato: msanto@fiocruz.br

\section{Antoine Metten}

É mestre em Sciences de Gouvernement Comparées pela Institut d’Étude Politique de Grenoble e membro do Grupo de Pesquisa de Inovação em Saúde da Fiocruz. Contato: antoine.metten@gmail.com / antoinemetten@fiocruz.br 